\title{
Primary Thyroid Lymphoma: an ominous and commonly forgotten association with Hashimoto's Thyroiditis
}

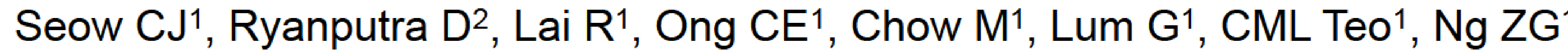 \\ 1Department of Endocrinology, Tan Tock Seng Hospital, Singapore \\ ${ }^{2}$ Yong Loo Lin School of Medicine, National University of Singapore, Singapore
}

Tan Tock Seng

\section{BACKGROUND}

Primary Thyroid Lymphoma (PTL) is rare and constitutes $2-5 \%$ of thyroid malignancies. The risk, however, increases with a background of Hashimoto's thyroiditis (HT). We report a patient with HT complicated by thyroid lymphoma and a literature review on this topic.

\section{CASE DESCRIPTION}

A 61 year-old Chinese lady with a background of Hashimoto's thyroiditis on levothyroxine therapy presented with an enlarging neck mass of 6 months duration. She experienced dysphagia for the last few weeks prior to the consultation but there were no other compressive symptoms such as shortness of breath or hoarseness of voice. There were no constitutional symptoms such as fever, chills, rigors or loss of weight.. She had no other significant medical history of note. There was no prior history of head and neck irradiation. There was no family history of malignancies. On examination, a large goiter was noted. There were no lymph nodes palpable. There was no organomegaly on abdominal palpation and the rest of the examination was unremarkable.

Investigation results: Free Thyroxine level 14 pmol/L (NR: 8-21); Thyroid Stimulating Hormone level 2.03 mIU/L (NR: 0.34-5.60); Thyroid Peroxidase Antibody $227 \mathrm{IU} / \mathrm{mL}(\mathrm{NR}: 5-34)$

An ultrasound of the thyroid gland (Figure 1) showed a lobulated heterogeneous mass measuring $5.5 \times 3.2 \times 2.9 \mathrm{~cm}$ with internal vascularity and suggestion of echogenic specks within. Computed Tomograpy of the neck done subsequently revealed a $6.7 \times 7.0 \times 4.1 \mathrm{~cm}$ mass (Figure 2 ). Infiltration of the postcricoid region is seen, together with left thyroarytenoid muscle involvement. There is also possible infiltration through the left lateral tracheal wall and possible encasement of the left common carotid artery.

An open biopsy of the thyroid mass was performed which revealed a diagnosis of Diffuse Large B-Cell Lymphoma (DLBCL). Bone marrow aspirate and trephine examination did not reveal any marrow involvement. She was referred to the medical oncologist and chemotherapy (R-CHOP) was initiated promptly. She responded well with considerable shrinkage of the neck mass.

\section{INVESTIGATIONS}

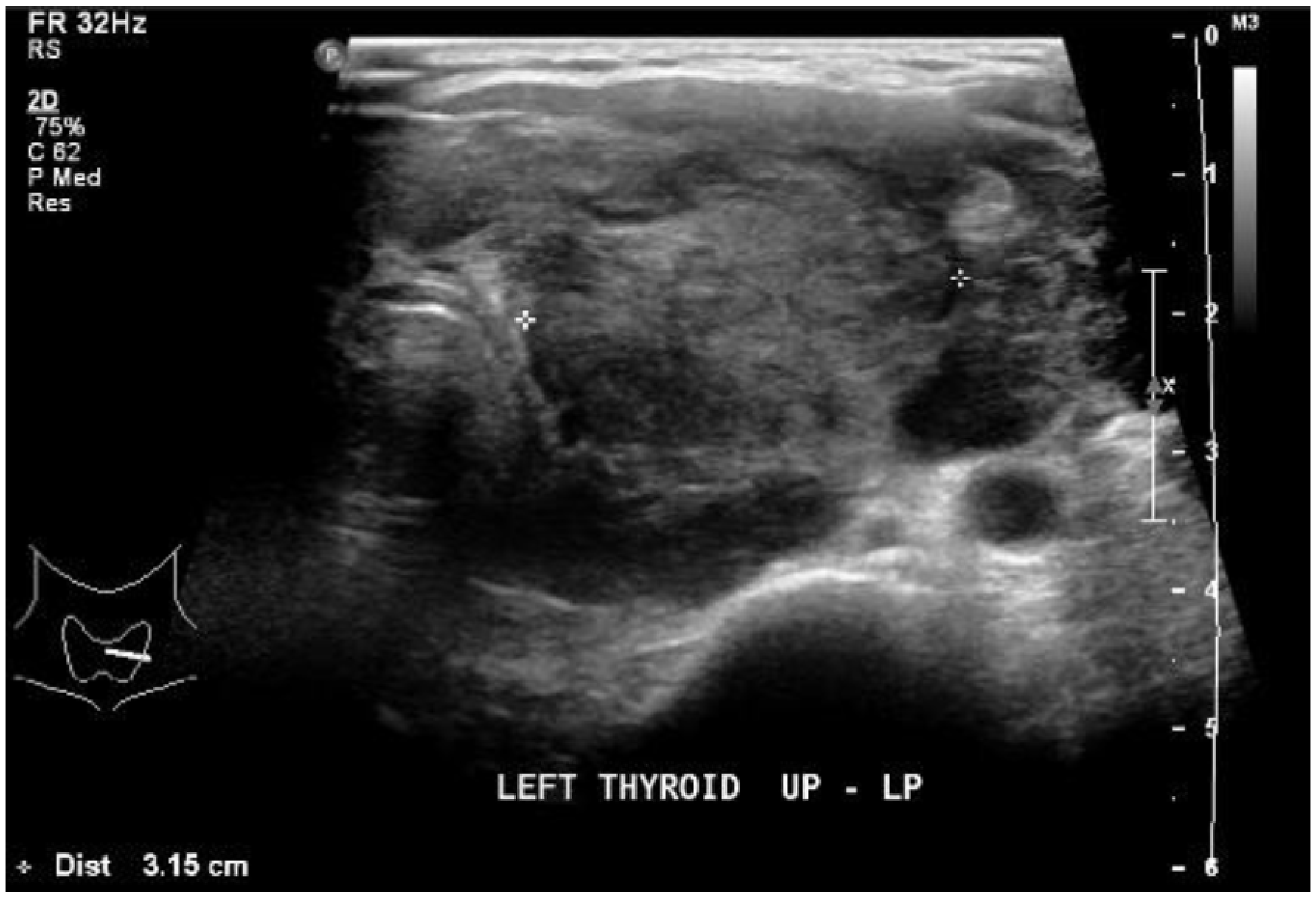

Figure 1: Ultrasound of the thyroid gland

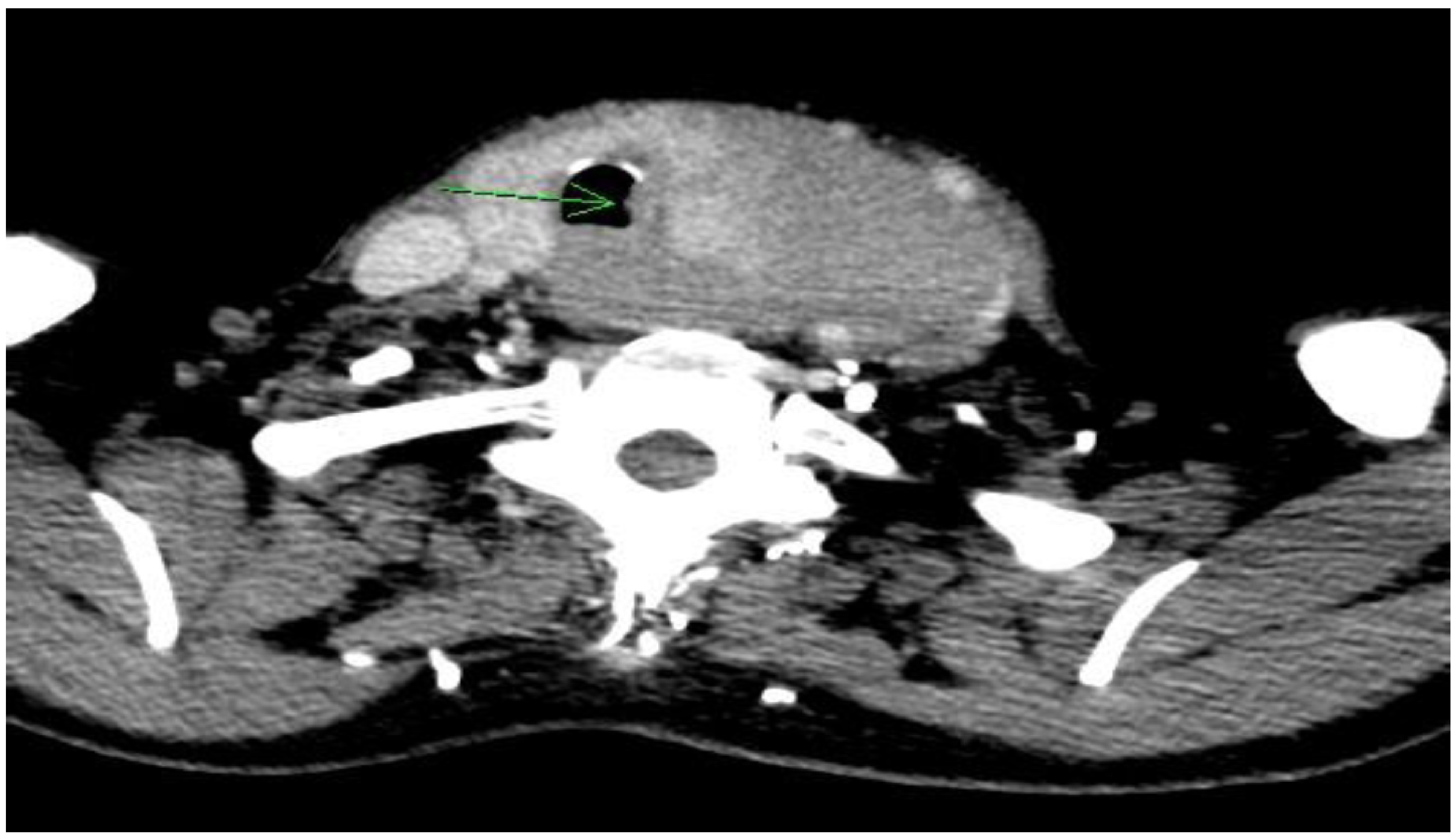

Figure 2: CT neck showing possible infiltration of the mass through the left lateral tracheal wall

\section{DISCUSSION}

PTL has an estimated annual incidence of two cases per million and almost all are of B-cell origin. It affects mainly middle-aged to older females. Most are diagnosed with Stage I disease and the most common histologic subtype is diffuse large B-cell lymphoma. It is estimated that the relative risk of developing a thyroid lymphoma is increased 67- to 80fold in patients with HT. Proposed mechanisms include prolonged antigenic stimulation in the setting of autoimmune thyroiditis leading to lymphomatous transformation or malfunction of the somatic hypermutation process, a phenomenon called aberrant somatic hypermutation process, which is regarded as a mechanism of lymphomagenesis. Treatment comprises different modalities depending on histologic subtype and stage of disease.

\section{CONCLUSION}

The diagnosis of primary thyroid lymphoma should be considered in a rapidly growing goiter especially in the background of HT. Early detection may result in improved survival

\section{REFERENCES}

Jayaprakash $\mathrm{K}$ et al. Hashimotos Thyroiditis with Coexistent Papillary Carcinoma and Non hodgkin Lymphoma-thyroid. Ann Med Health Sci Res. 2014 Mar;4(2):268-70

2. Nam YJ et al. Co-occurrence of Papillary Thyroid Carcinoma and Mucosa-Associated Lymphoid Tissue Lymphoma in a patient with long-standing Hashimoto Thyroiditis. Endocrinol Metab (Seoul) 2013 Dec;28(4):341-5

Vassilatou $\mathrm{E}$ et al. Coexistence of differentiated thyroid carcinoma with primary thyroid lymphoma in a background of hashimoto thyroiditis. J Clin Onco 2011 Sep 1;29(25):e70912 